\title{
Role of tacrolimus prolonged release in the prevention of allograft rejection
}

This article was published in the following Dove Press journal:

Transplant Research and Risk Management

6 August 2010

Number of times this article has been viewed

\section{Peter Abrams \\ Abhinav Humar \\ Henkie P Tan}

Department of Surgery, Thomas E Starzl Transplantation Institute, University of Pittsburgh School of Medicine, Pennsylvania, USA
Correspondence: Henkie P Tan

Department of Surgery, Thomas E Starzl Transplantation Institute, University of Pittsburgh School of Medicine, UPMC Montefiore, 7 South, 3459 Fifth Avenue, Pittsburgh, PA 15213, USA

Email tanhp@upmc.edu
Abstract: Successful management of the solid-organ transplant recipient begins with prevention of rejection and achieving a balance between insufficient and excessive immunosuppression. Standard tacrolimus therapy for prevention of solid-organ transplant rejection consists of 2 divided doses per day. In an effort to simplify tacrolimus dosing to once daily, a new formulation (tacrolimus prolonged release [PR]) was chosen for its combination of a similar extent of bioavailability and a substantially reduced rate of clearance. Several clinical conversion studies have now been completed using PR to clarify its pharmacokinetics, efficacy at prevention of allograft rejection, and safety profiles in solid-organ transplant patients. A cohort of 67 stable kidney transplant recipients was converted from standard tacrolimus to PR in an open-label, multicenter study in the United States and Canada. A second open-label, multicenter study was performed in liver transplant recipients with stable graft function on standard tacrolimus therapy converted to PR. A third conversion study was performed as an open-label study at 5 centers in the United States in stable pediatric liver transplant recipients. As medication noncompliance can significantly contribute to the incidence of graft rejection and graft loss in transplant recipients, a potentially significant advance in the transplant community's ongoing mission to optimize prevention of rejection occurred with the development of a once-daily tacrolimus PR. The results of these preliminary studies suggest that select solid-organ transplant recipients converted to PR can be safely maintained using the same monitoring and patient care techniques historically used for standard tacrolimus therapy.

Keywords: immunosuppression, tacrolimus, allograft, organ transplant, rejection

\section{Preventing transplant organ rejection}

Preventing rejection of solid-organ transplants requires steadfast commitment and remains a lifelong endeavor on the part of the transplant recipient and the transplant management team. Made possible through the use of antirejection or immunosuppressive medications, the process of rejection prevention is successful only $50 \%$ overall for various reasons including drug-resistant immune-mediated chronic rejection pathways, the development of life-threatening opportunistic infections or malignancy due to excessive immunosuppression, and serious adverse drug reactions. In general, episodes of rejection can be treated successfully by a growing repertoire of potent immunosuppressants. However, escalation of immunosuppression to treat rejection can induce significant patient debilitation involving significant neurotoxicity, electrolyte disturbances, myelosuppression, and even organ failure. Therefore, successful management of the transplant recipient fundamentally begins with prevention of rejection, achieving that fine balance between too little and too 
much immunosuppression, fully understanding the unique circumstances of each individual patient, and successfully manipulating the cellular mechanisms that facilitate or oppose graft tolerance.

\section{Brief history of tacrolimus}

The discovery of tacrolimus in 1984, on Mount Tsukuba just north of Tokyo, Japan, led to the beginning of a new era in solid-organ transplantation. Initially recognized as a potent in vitro immunosuppressive agent, tacrolimus was found to inhibit interleukin-2 (IL-2) production associated with T-lymphocyte activation, resulting in the suppression of differentiation and proliferation of cytotoxic $\mathrm{T}$ cells. The transplant community was first made aware of the immunosuppressive properties of tacrolimus by researchers from Japan at the 11th World Congress of the Transplantation Society in Helsinki, Finland, in 1986. Further research regarding tacrolimus safety and efficacy was begun at the University of Pittsburgh. The first clinical trial of tacrolimus was performed as rescue therapy for liver transplant patients facing retransplantation or significant drug toxicity to treat ongoing severe organ rejection. The promising results in terms of patient and graft survival were presented at the 1990 Congress of the Transplantation Society in San Francisco, California, USA. The first trial using tacrolimus as firstline therapy was begun at the University of Pittsburgh in the spring of $1990 .{ }^{1}$ Two prospective, randomized trials conducted in the United States and Europe subsequently demonstrated that patient and liver graft survival were comparable between tacrolimus and cyclosporine; however, the rates of acute, steroid-resistant, and refractory rejection were significantly lower with tacrolimus therapy.,3 On the basis of these studies, tacrolimus was clinically introduced first in Japan in 1993 and subsequently in the United States in 1994. In 2003, nearly $90 \%$ of new liver transplant recipients and $67 \%$ of new kidney transplant recipients were discharged on tacrolimus-based immunosuppression. ${ }^{4,5}$

\section{Mechanism of action}

The activity of tacrolimus begins with its binding to an intracellular protein, FKBP-12. This initial event leads to the formation of a complex of tacrolimus-FKBP-12, calcium, calmodulin, and calcineurin, which inhibits the phosphatase function of calcineurin. Inhibition of calcineurin prevents the dephosphorylation and nuclear translocation of the nuclear factor of activated T-lymphocytes, both of which are critical events in the initiation of gene transcription for the production of IL-2 and other lymphokines. By blocking production of IL-2 and other lymphokines through calcineurin inhibition, tacrolimus potently suppresses T-lymphocyte activation, resulting in clinical immunosuppression and prevention of organ rejection.

Tacrolimus is rapidly absorbed in the gastrointestinal tract. Peak serum concentrations of tacrolimus after oral administration occur in approximately 1-2 hours. Tacrolimus as a compound is almost completely metabolized through the cytochrome (CYP) P450 hepatic pathway before elimination. Additional metabolism of tacrolimus occurs by CYP3A4 isoenzymes and P-glycoprotein in the intestinal mucosa. ${ }^{6,7}$

\section{Advances in tacrolimus dosing}

Standard tacrolimus therapy (TAC) for prevention of solid-organ transplant rejection consists of 2 divided doses per day at dosages sufficient to maintain whole-blood trough levels in the range of 5-15 $\mathrm{ng} / \mathrm{mL}$. In an effort to simplify tacrolimus dosing to once daily, a new formulation (MR-4, herein referred to as prolonged release $[\mathrm{PR}]$ ) was chosen for its combination of a similar extent of bioavailability to the original version of tacrolimus and a substantially reduced rate of clearance. This drug profile was achieved through modifications of the drug capsule without altering the drug compound itself. PR was initially administered to over 100 healthy human volunteers in 5 unpublished phase 1 studies ( 3 single-dose studies in the United States and 2 repeat-dose studies in Europe). The PR formulation was reportedly well tolerated in all phase 1 studies. The repeat-dose studies demonstrated that the 24-hour measured blood concentrations or bioavailability of PR were approximately equal to the sum of the 12-hour measured blood concentrations of twice-daily standard tacrolimus, suggesting these drug formulations were bioequivalent. ${ }^{8}$

Several clinical conversion studies have now been completed using PR in an effort to clarify and elaborate its pharmacokinetics (PK) in solid-organ transplant patients. Alloway et $\mathrm{al}^{9}$ evaluated the conversion PK of 67 stable kidney transplant recipients converted from standard tacrolimus to PR in an open-label, multicenter, single-sequence, crossover study conducted in the United States and Canada with patient ages ranging from 18 to 65 years. For 1 week, patients were administered their established dose of tacrolimus and underwent routine drug concentration testing at the beginning and end of that week. These patients were then converted to the same 
milligram-for-milligram daily dose of PR in the morning on day 8. Similar drug measurements were recorded at day 8, 14, and 21 while taking once-daily PR. With day 8 , PK data not included in the analysis, the measured blood concentrations were comparable, supporting a 1:1 conversion of tacrolimus twice-daily to PR once-daily.

A second PK study of PR in transplant patients involved an open-label multicenter PK study in liver transplant recipients with stable graft function on standard tacrolimus therapy converted to PR. ${ }^{10}$ The study population included 69 patients from 10 centers in the United States with an age range from 18 to 65 years, who had undergone liver transplant at least 6 months prior to enrollment and were receiving stable doses of tacrolimus ( $>2$ weeks) with a serum creatinine level $<2.0 \mathrm{mg} / \mathrm{dL}$ before enrollment. Patients were converted back and forth from standard tacrolimus to PR once-daily in the morning in 2-week intervals in a single sequence, 4-period crossover study design. Patients were converted to the same milligram-for-milligram daily dose of PR daily on days 15-28 and days 43-56. Twenty-four-hour PK profiles were obtained on days 14, 28, 52, and 56. The measured blood concentrations of tacrolimus and PR again indicated that at steady state, PR was relatively equivalent to standard tacrolimus after a milligram-for-milligram conversion, this time in stable liver transplant recipients.

A third conversion study was performed as an open-label study at 5 centers in the United States in stable pediatric liver transplant recipients. ${ }^{11} \mathrm{PK}$, as well as safety and tolerability of $\mathrm{PR}$, was evaluated in 18 patients $\leq 12$ years of age, who were receiving a stable tacrolimus-based immunosuppression regimen for at least 2 weeks. Similar to previous studies, patients were converted to PR on a 1:1 (mg:mg) basis for their total daily dose, with serial collection of whole-blood samples for PK profiles. Exposure to tacrolimus at steady state was once again reported to be equivalent between PR and standard tacrolimus.

\section{Efficacy of PR tacrolimus}

In addition to PK data, these conversion studies also evaluated the efficacy and long-term safety profiles of PR in comparison to standard tacrolimus therapy in the same patient cohorts. Clinical efficacy data at 2-year postconversion for the stable adult kidney and liver transplant recipients were published in 2007. 12,13 Two-years postconversion follow-up was achieved in 59 of the original 67 kidney transplant patients. The mean posttransplant time period prior to PR therapy conversion was 3.8 years (range, $0.64-11.13$ years).
The mean tacrolimus whole-blood level ranged from 5.7 to $7.1 \mathrm{ng} / \mathrm{mL}$ in the 2-year postconversion period. Of the 57 kidney transplant recipients receiving mycophenolate mofetil (MMF) prior to study enrollment, 54 recipients continued on MMF therapy. Six recipients were receiving azathioprine (AZA) prior to enrollment, and no reported changes in AZA were reported during follow-up. Of the original 67 recipients who participated in the conversion study, 61 patients were taking steroids prior to enrollment, 10 of whom required an increase in steroid dosage during follow-up ( 5 for maintenance therapy at the discretion of the investigator, 4 for treatment of rejection, and 1 for vasculitis). Steroid use was decreased in 16 recipients (15 for maintenance therapy at the discretion of the investigator and 1 postrejection therapy at the discretion of the investigator). Patient and graft survival at 2-year postconversion were $100 \%$ (67 of 67) and 98.5\% (66 of 67), respectively, and the incidence of biopsy-confirmed acute rejection (BCAR) was $6.0 \%$. The 4 episodes of acute rejection (grade IA, IB, IIA, and IIB) occurred in 4 different patients at 719, 227, 155, and 458 days, respectively, after conversion to PR. At the time of diagnosis of BCAR, tacrolimus levels were between 5 and $12 \mathrm{ng} / \mathrm{mL}$ for all but 1 recipient, who had a trough level of $3.0 \mathrm{ng} / \mathrm{mL}$ measured approximately 48 hours preceding the diagnosis of acute rejection.

In the conversion study involving stable adult liver transplant recipients, complete follow-up data were achieved in 56 of 69 patients on PR treatment for 2 years. The mean posttransplant time interval prior to PR conversion was also 3.8 years (range $0.82-12.4$ years). The mean tacrolimus whole-blood level ranged from 6.2 to $6.6 \mathrm{ng} /$ $\mathrm{mL}$ over the 2-year postconversion. Prior to enrollment, 22 patients were taking MMF, 2 patients were taking AZA, and 41 patients were on steroid therapy. These cotherapies were not altered during the 2-year postconversion period. The reported incidence of BCAR at 2-year postconversion was $5.8 \%$. Rejection episodes included 2 grade I rejections, and 1 episode each of grade II and grade III rejection according to standard Banff criteria. These BCAR episodes occurred at 190, 110, 357, and 10 days postconversion, respectively. All 4 episodes of BCAR were steroid treatment responsive, and there were no reports of multiple rejections in a single patient. Patient and graft survival at 2-year postconversion was $98.6 \%$. One white female reportedly died with a functioning graft due to complications from squamous cell lung cancer nearly 22 months after conversion to PR. ${ }^{13}$ 
In the pediatric liver transplant conversion study, an analysis of drug efficacy at 1 -year postconversion was performed in all 18 patients. In this group of stable pediatric liver transplant recipients, the mean posttransplant time interval prior to conversion to PR was 4 years. Mean whole-blood levels of tacrolimus were between 4.9 and $5.9 \mathrm{ng} / \mathrm{mL}$ during the first 12 months postconversion to PR. All adjuvant immunosuppression was maintained without alteration throughout the first 14 days of the study, subsequent to which adjustments were permitted. Of the 18 patients, 7 patients receiving PR did not require any change in dose during the 1-year postconversion and 5 patients required only a single dose adjustment. A total of 14 patients were receiving adjunctive immunosuppression prior to study enrollment, and most patients were continued throughout the course of the study. It is important to note that patients were excluded from this study if they had experienced any rejection episode within 90 days prior to enrollment, any rejection episode within 6 months requiring antilymphocyte antibody therapy, or more than 2 rejection episodes within the last 12 months. At 1-year postconversion to PR, there were no cases of BCAR, discontinuation of PR therapy, and graft loss or death.

Most recently, a large phase 3, randomized (1:1:1), open-label, 3-arm (PR/MMF, TAC/MMF, and cyclosporine [CsA]/MMF) noninferiority study was conducted in 60 centers in the United States, Canada, and Brazil, in de novo kidney transplant recipients. ${ }^{14}$ The dosing of the 3 primary immunosuppressants was chosen on the basis of recommended ranges and was adjusted on the basis of protocol-specified target whole-blood trough levels and according to standard clinical practice. Two doses of basiliximab induction therapy were administered to all patients on day 0 and between days 3 and 5. Patients were also maintained on corticosteroids, initiated on day 0 (500-1000 mg methylprednisolone or equivalent intravenous bolus) followed by oral administration of $200 \mathrm{mg}$ methylprednisolone (or equivalent) on day 1 and subsequent tapering to a mean prednisone equivalent of $5-10 \mathrm{mg} / \mathrm{d}$ after 3 months. MMF ( $1 \mathrm{~g}$ twice a day) was administered according to package insert guidelines, and up to $1.5 \mathrm{~g}$ twice a day was permitted in black patients. Target levels for mycophenolic acid were not standardized in the study protocol. Thirty of 668 randomized patients did not receive study drug; of the remainder, 214 patients were randomized to $\mathrm{PR} / \mathrm{MMF}$, 212 to TAC/MMF, and 212 to CsA/MMF. Treatment groups were balanced with regard to donor type and other baseline characteristics. Eighty-five percent of patients in the PR/MMF and TAC/MMF groups completed 1 year of randomized treatment compared with $71 \%$ of patients in the CsA/MMF group.

At 1-year posttransplant, the open-label study found no statistical differences in the Kaplan-Meier estimates for patient and graft survival among all 3 treatment groups. Of note, no statistical significance was reported in evaluating the differences seen in patient and graft survival as well as the number and treatment of BCAR between the 2 tacrolimus groups in particular. The incidence of BCAR at 6 months and 1 year was significantly lower in the TAC/MMF group than in the CsA/MMF group. No statistically significant differences were observed between the PR/MMF and CsA/MMF groups in terms of incidence of BCAR at 6 months and 1 year. The incidence of BCAR at 1 year in patients who received grafts from deceased donors was significantly lower $(P \leq 0.015)$ in the PR/MMF (10/111, 9.0\%) and TAC/MMF (8/106, 7.5\%) groups than in the CsA/MMF group (21/101, 20.8\%).

\section{Safety of prolonged release tacrolimus}

In the stable kidney transplant recipient conversion study, the incidence of posttransplant diabetes mellitus, hyperlipidemia, hypertension, infectious episodes, renal dysfunction, or hepatic dysfunction was similar to rates observed in previous studies with standard tacrolimus therapy. Serious adverse events reported over the 2-year postconversion period included cellulitis (4.5\%), human polyomavirus $(3.0 \%)$, pyelonephritis $(3.0 \%)$, urinary tract infection $(3.0 \%)$, increased serum creatinine $(3.0 \%)$, and acute renal failure $(4.5 \%)$. The clinical laboratory profile, including serum glucose, blood urea nitrogen, serum creatinine, creatinine clearance, serum aspartate aminotransferase, alanine aminotransferase, alkaline phosphatase, total bilirubin, and serum cholesterol, was within normal limits and remained stable in the 2-year postconversion period. Increased isolated fasting plasma glucose $(\geq 126 \mathrm{mg} / \mathrm{dL})$ was observed in $21.2 \%$ of recipients. No cases of new-onset insulin or oral hypoglycemic agent use or of glycosylated hemoglobin $\geq 6 \%$ during the 2 -year postconversion period were reported.

In the stable adult liver transplant conversion study, the safety profile of PR was consistent with that previously reported for standard tacrolimus therapy. ${ }^{15-18}$ Compared with historical rates with standard tacrolimus dosing, the authors found no increased risk of infection, renal dysfunction, hepatic dysfunction, posttransplant diabetes mellitus, hyperlipidemia, or hypertension in the postconversion 
period. Serious adverse events included pyrexia (5.8\%), incisional hernia $(5.8 \%)$, cholangitis $(2.9 \%)$, cholestasis $(2.9 \%)$, diarrhea $(2.9 \%)$, influenza $(2.9 \%)$, and pneumonia (2.9\%). There were no significant differences between liver transplant recipients who were receiving MMF and/or AZA cotherapy and those who were not in terms of the 2-year incidence of renal, hepatic, or lipid-related adverse events, hypertension, gastroenteritis, gastrointestinal disturbances, or glucose abnormalities. This was also true of patients taking steroid cotherapy compared with those patients on steroid-free regimens. However, significantly $(P=0.0179)$ more patients who took prednisone demonstrated glucose abnormalities than those who did not. Approximately, $23 \%$ of patients had new-onset fasting plasma glucose levels $\geq 126 \mathrm{mg} / \mathrm{dL}$ during the 2 years after conversion. A reported $4.7 \%$ of patients had new-onset requirement for insulin, whereas $4.7 \%$ had new-onset requirement for an oral hypoglycemic medication.

In the pediatric liver transplant conversion study, safety of PR was assessed on the basis of adverse events, and the results of routine clinical laboratory tests and vital sign measurements were collected according to protocol. The postconversion safety profile of PR in the pediatric liver transplant study was similar to the well-established profile for patients maintained on standard tacrolimus therapy. No new cases of diabetes mellitus or glucose metabolism disorder were reported. There was no reported trend to suggest an increase in the incidence of adverse events over the duration of PR therapy. No de novo malignancies were detected during the 1-year postconversion follow-up in this patient population.

In the de novo kidney transplant study, the safety profile of $\mathrm{PR} / \mathrm{MMF}$ in comparison with $\mathrm{CsA} / \mathrm{MMF}$ was similar to the observed TAC/MMF profile and consistent with previously reported profiles of TAC in comparison with CsA. There were no significant differences in rates of bacterial, fungal, or viral infections between treatment groups. Consistent with previous reports, the incidence of new-onset oral hypoglycemic agent use was significantly higher in both tacrolimus groups compared with the CsA/ MMF group. However, the incidence of new-onset use of insulin $\geq 30$ days was not significantly different between treatment groups.

\section{Conclusions}

As medication noncompliance can be a significant variable contributing to the incidence of graft rejection and graft loss in transplant recipients, a potentially significant advance in the transplant community's ongoing mission to optimize prevention of rejection occurred with the development of a once-daily tacrolimus PR. Although there is no direct evidence that the use of PR may improve compliance in comparison to TAC, keeping in mind the reasons for antirejection medication noncompliance are complex, it is well established that reducing the dosing frequency of medications can be a significant contributor to improving compliance. ${ }^{19,20}$ The results of these preliminary studies suggest that select solid-organ transplant recipients converted to PR can be safely maintained using the same therapeutic monitoring and patient care techniques historically used for standard tacrolimus.

\section{Disclosure}

The authors report no conflicts of interest in this work.

\section{References}

1. Todo S, Fung JJ, Starzl TE, et al. Liver, kidney and thoracic organ transplantation under FK 506. Ann Surg. 1990;212:295.

2. The US Multicenter FK506 Liver Study Group. A comparison of tacrolimus (FK 506) and cyclosporine for immunosuppression in liver transplantation. N Engl J Med. 1994;331:1110.

3. European FK506 Multicentre Liver Study Group. Randomised trial comparing tacrolimus (FK 506) and cyclosporin in prevention of liver allograft rejection. Lancet. 1994;344:423.

4. UNOS Market Share Report, Feb 2004.

5. Fung JJ. Tacrolimus and transplantation: a decade in review. Transplantation. 2004;77:S41-S43.

6. Prograf [package insert]. Deerfield, IL: Fujisawa Healthcare Inc; 2003.

7. Christians U, Jacobsen W, Benet LZ, et al. Mechanisms of clinically relevant drug interactions associated with tacrolimus. Clin Pharmacokinet. 2002;41(11):813.

8. First MR, Fitzsimmons WE. New drugs to improve transplant outcomes. Transplantation. 2004;77:S88-S92.

9. Alloway R, Steinberg S, Khalil K, et al. Conversion of stable kidney transplant recipients from a twice-daily prograf-based regimen to a once-daily modified release tacrolimus-based regimen. Transplant Proc. 2005;37:867-870.

10. Florman S, Alloway R, Kalayoglu M, et al. Conversion of stable liver transplant recipients from a twice-daily prograf-based regimen to a once-daily modified release tacrolimus-based regimen. Transplant Proc. 2005;37:1211-1213.

11. Heffron TG, Pescovitz MD, Florman S, et al. Once-daily tacrolimus extended-release formulation: 1-year post-conversion in stable pediatric liver transplant recipients. Am J Transplant. 2007;7: 1609-1615.

12. Alloway R, Steinberg S, Khalil K, et al. Two years postconversion from a prograf-based regimen to a once-daily tacrolimus extended-release formulation in stable kidney transplant recipients. Transplantation. 2007;83:1648-1651

13. Florman S, Alloway R, Kalayoglu M, et al. Once-daily tacrolimus extended release formulation: experience at 2 years postconversion from a prograf-based regimen in stable liver transplant recipients. Transplantation. 2007;83:1639-1642.

14. Silva HT, Yang HC, Abouljoud M, et al. One-year results with extended-release Tacrolimus/MMF, tacrolimus/MMF and cyclosporine/ MMF in de novo kidney transplant recipients. Am J Transplant. 2007; 7:595-608. 
15. Knoll GA, Bell RC. Tacrolimus versus cyclosporine for immunosuppression in renal transplantation: meta-analysis of randomized trials. BMJ. 1999;318:1104-1107.

16. McAlister VC, Haddad E, Renouf E, et al. Cyclosporine versus tacrolimus as primary immunosuppressant after liver transplantation: a meta-analysis. Am J Transplant. 2006;6:1578-1585.

17. Kobashigawa JA, Miller LW, Russell SD, et al. Tacrolimus with mycophenolate mofetil (MMF) or sirolimus vs cyclosporine with MMF in cardiac transplant patients: 1-year report. Am J Transplant. 2006; 6:1377-1386.
18. Grimm M, Rinaldi M, Yonan NA, et al. Superior prevention of acute rejection by tacrolimus vs cyclosporine in heart transplant recipients a large European trial. Am J Transplant. 2006;6:1387-1397.

19. Eisen SA, Miller DK, Woodward RS, et al. The effect of prescribed daily dose frequency on patient medication compliance. Arch Intern Med. 1990;150:1881-1884.

20. Osterberg L, Blaschke T. Adherence to medication. $N$ Engl J Med. 2005;353:487-497.

\section{Publish your work in this journal}

Transplant Research and Risk Management is an international, peerreviewed open access journal focusing on all aspects of transplantation and risk management to achieve optimal outcomes in the recipient improving survival and quality of life. The journal welcomes submitted papers covering original research, basic science, clinical studies, reviews \& evaluations, guidelines, expert opinion and commentary, case reports and extended reports. The manuscript management system is completely online and includes a very quick and fair peer-review system, which is all easy to use. Visit http://www.dovepress.com/ testimonials.php to read real quotes from published authors. 